\title{
Evaluation of histomorphological findings and malignancies in postmenopausal bleeding
}

\author{
M. Pavani ${ }^{1, *}$, Ch. Geetha ${ }^{2}$, L. Peter Ericson ${ }^{3}$, Ashok Kumar Deshpande ${ }^{4}$ \\ ${ }^{1,2}$ Assistant Professor, ${ }^{3}$ Associate Professor, ${ }^{4}$ Professor and HOD, ${ }^{1,2,4}$ Dept. of Pathology, ${ }^{3}$ Dept. of Anatomy, Kamineni \\ Academy of Medical Sciences \& Research Centre, Hyderabad, India
}

*Corresponding Author:

Email: pavanipeter@gmail.com

\begin{abstract}
Introduction: In this era, as the life expectancy is increasing, women are experiencing a long postmenopausal phase. Postmenopausal bleeding (PMB) is a sinister complaint and accounts for about $10 \%$ of postmenopausal complaints. Being a symptom of varied etiologies and its strong association with malignancy, it should be diligently evaluated.

Aims and Objectives: 1 . To evaluate the histopathological spectrum of lesions presenting as PMB. 2. To estimate the incidence of premalignant, and malignant lesions presenting as PMB. 3. To confirm the benign nature of the lesions to prevent unnecessary radical treatment.

Materials and Methods: This prospective study done from January 2016 to September 2017 and included the endometrial biopsies/curettages and cervical biopsies sent for histopathological examination from clinically diagnosed cases of postmenopausal bleeding.

Results: Out of all the samples majority were benign lesions (44\%), followed by premalignant (25.7\%), malignant lesions $(18.3 \%)$.

Conclusion: A variety of histopathological findings are seen in PMB.

Benign pathologies were more common in our study but the collective proportion of premalignant and malignant lesions is considerably higher in this study.

Accurate diagnosis makes it much easier to counsel the patient about further course of management, prevent unnecessary radical treatment for benign conditions, prompt treatment, and vigilant follow up for malignant lesions.
\end{abstract}

Keywords: Postmenopausal bleeding (PMB), Endometrial carcinoma, Histopathology.

\section{Introduction}

Menopause is a physiological event in women signalling the end of the reproductive years and is associated with signs of estrogen deficiency. Menopause is derived from the Greek word meno, month and pause to stop. ${ }^{1}$ Postmenopause is a challenging period for many women and has a considerable impact on women's health related quality of life. ${ }^{2}$ Average age at menopause in Indians is around 45 to 50 years. ${ }^{3}$

WHO has defined postmenopausal bleeding (PMB) as an episode of bleeding twelve months or more after the last menstrual period resulting from loss of ovarian activity. ${ }^{3}$ Seventy percent of cases of postmenopausal bleeding have benign etiology but the remaining $30 \%$ are associated with malignancy. ${ }^{4}$ The presentation of PMB may be as heavy bleeding, minimal bleeding or just spotting. ${ }^{5}$

Postmenopausal bleeding is as an ominous and serious alarm of genital pathologies and classic medical teaching has labelled postmenopausal bleeding as "Endometrial cancer until proven otherwise". 6 Postmenopausal Bleeding (PMB) is a common clinical problem and occurs in approximately $3 \%$ of postmenopausal women. ${ }^{7}$
Postmenopausal bleeding occurs over a wide age range and can result from a number of pathologies arising in any of the female genital tract organs, particularly uterus and cervix. ${ }^{8}$ PMB can result from the normal physiologic atrophic changes, infections, benign lesions such as polyps, hyperplastic (pre-malignant) conditions and aggressive malignant conditions. A number of systemic disorders, especially blood dyscrasias are also associated with this symptom. ${ }^{9}$

There is an increasing trend towards uterine malignancies in the past two decades. Hence postmenopausal bleeding requires a thorough evaluation clinically and pathologically. ${ }^{4}$ In developed countries the risk of endometrial cancer in postmenopausal bleeding women is high whereas risk of cervical cancer is higher in developing countries. ${ }^{10}$ Endometrial and cervical cancers if detected early, can be cured completely.

Endometrial hyperplasia is one of the most important predisposing factors for the development of endometrial carcinoma. ${ }^{1} \quad$ Atypical endometrial hyperplasia has a higher risk of malignant transformation than with endometrial hyperplasia without atypia.

Cervical cancer is one of the common malignancies presenting as PMB in developing countries. 
Premalignant and malignant lesions of the cervix may also present as postmenopausal bleeding. ${ }^{11}$

Due to lack of public awareness, screening programmes, and inadequate health facilities, this symptom is usually ignored along with other medical problems and the women present at a very late stage

PMB continues to be a huge burden on the healthcare system with varying etiology and considerable social implications. Therefore, this threatening medical situation necessitates the estimation of the recent disease burden. ${ }^{9}$ World wide a number of studies on PMB have been conducted, but there are few reports from India especially from Telangana region of South India.

The current study was carried out to evaluate various causes of postmenopausal bleeding based on histopathology, and the percentage of various benign, premalignant and malignant lesions in post menopausal bleeding.

\section{Aims and Objectives}

1. To evaluate the histopathological spectrum of various lesions.

2. To detect the premalignant lesions so that accurate treatment may be given promptly.

3. To find the incidence of malignant lesions presenting as PMB

4. To confirm the benign nature of the lesions to prevent unnecessary radical treatment.

\section{Materials and Methods}

The present study is a prospective study conducted in the department of Pathology from January 2016 to September 2017. The material for the study was collected from the endometrial biopsies/curettages and cervical biopsies sent for histopathological examination from clinically diagnosed cases of postmenopausal bleeding. In few of the cases simultaneous endometrial and cervical biopsies were done.

Hysterectomy specimens were not included in the study. The endometrial curettage and cervical biopsy material was immediately fixed in $10 \%$ fresh formalin. Routine processing was done and slides were stained with Haematoxylin \& Eosin (H\&E). Histopathological evaluation was done by two pathologists and the causes for postmenopausal bleeding were evaluated.

\section{Inclusion Criteria}

Post-menopausal women with complaints of per vaginal bleeding.

\section{Exclusion Criteria}

1. Patients with bleeding disorders / blood dyscrasias.

2. Patients on anticoagulant therapy

3. Premature menopause whether surgical or natural

4. Age less than 40 years

5. Patients on hormone replacement therapy.
The history of postmenopausal bleeding varied from spotting per vagina, brownish discharge, scanty flow and moderate to profuse bleeding, presenting six months or more after menopause.

The study group included 102 patients out of which seven patients had both endometrial and endocervical biopsies. A total of 109 biopsy samples were included in the study group.

\section{Results}

A total of 109 biopsy /curettage specimens were included in this study from 102 patients. The age of the women ranged from 41 years to 84 years and majority of the women were between 51 to 60 years of age. (Table 1) The incidence of malignancy was more in elderly age group and the number of cases with postmenopausal bleeding decreased with age.

Table 1: Frequency of cases in different age groups

\begin{tabular}{|l|c|c|}
\hline S.No & Age (Yrs) & No: of cases \\
\hline 1 & $40-50$ & 23 \\
\hline 2 & $51-60$ & 40 \\
\hline 3 & $61-70$ & 24 \\
\hline 4 & $71-80$ & 14 \\
\hline 5 & $81-$ above & 01 \\
\hline
\end{tabular}

Out of the 109 specimens majority were endometrial biopsies followed by cervical biopsies and curettage samples. (Table 2) We had a spectrum of lesions comprising of inflammatory lesions, benign lesions, premalignant and malignant lesions. Out of all the samples majority were diagnosed as benign lesions $(44 \%)$, premalignant $(25.7 \%)$, followed by malignant lesions $(18.3 \%)$. Inadequate samples (11.9\%) were also encountered in our study. (Chart 1)

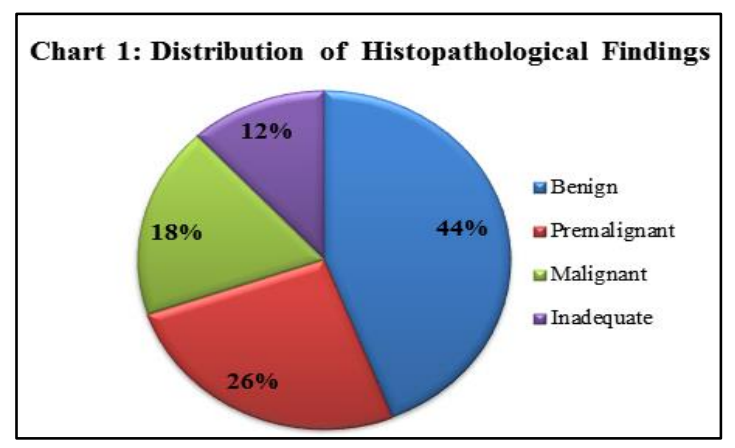

Table 2: Percentage of various biopsy specimens received

\begin{tabular}{|l|c|c|c|}
\hline S.No & Sample & $\begin{array}{c}\text { No: of } \\
\text { Samples }\end{array}$ & $\%$ \\
\hline 1 & $\begin{array}{c}\text { Endometrial } \\
\text { Biopsy }\end{array}$ & 71 & $65.14 \%$ \\
\hline 2 & $\begin{array}{c}\text { Endometrial } \\
\text { curettage }\end{array}$ & 03 & $2.75 \%$ \\
\hline
\end{tabular}




\begin{tabular}{|l|c|c|c|}
\hline 3 & Cervical Biopsy & 35 & $32.11 \%$ \\
\hline 4 & Total & 109 & $100 \%$ \\
\hline
\end{tabular}

Benign Lesions: Endocervical polyps were more in number followed by endometrial polyps, and proliferative type of endometrium. Eleven percentage of the cases showed atrophic endometrium. (Chart 2)

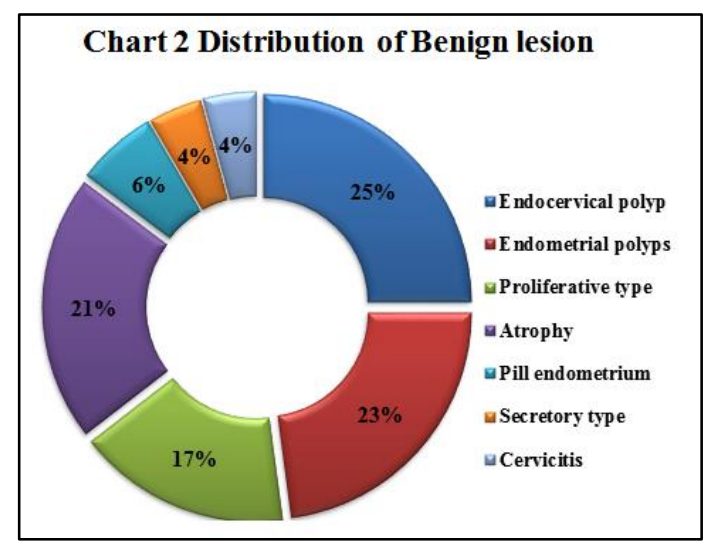

Chart 3 Distribution of premalignant lesions
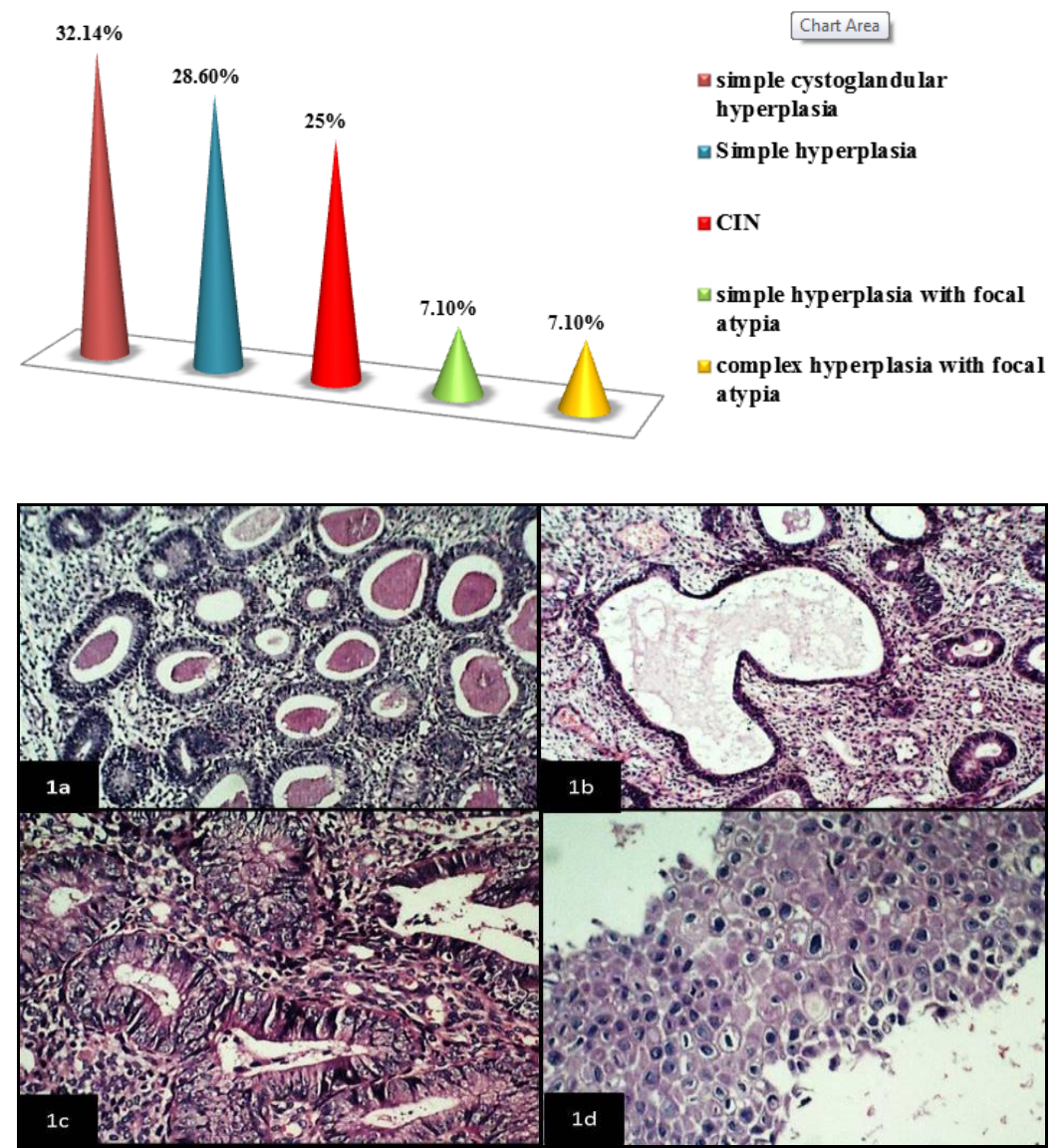

Fig. 1: Fig. 1a: Low power view, H/E - Simple hyperplasia

Fig. 1b: Low power view, H/E - Cystic glandular hyperplasia

Fig. 1c: High power view, H/E- Simple hyperplasia with focal atypia

Fig.1d: Low power view, H/E- Cervical intra epithelial neoplasia III 
Malignant Lesions: In our study we observed $18.34 \%$ (20 cases) of malignant lesions. The age of presentation of maximum number of malignant lesions was between 51 to 60 years. Out of the 20 cases $70 \%$ were reported mixed mullerian tumor. In the endometrial carcinomas well differentiated endometroid type of endometrial carcinomas were maximum. In cervical carcinomas well differentiated squamous cell carcinomas were more in number (Table 3. Fig. 2)

as endometrial carcinomas, $25 \%$ were cervical carcinomas and we had one case $(5 \%)$ of malignant

Table 3: Distribution of various malignant lesions

\begin{tabular}{|l|c|c|c|}
\hline S.No & Malignant lesion & No: of lesions & \% \\
\hline \multirow{3}{*}{1.} & Endometrial carcinoma & 14 & $70 \%$ \\
\cline { 2 - 4 } & Well differentiated endometrioid type & 10 & $50 \%$ \\
\cline { 2 - 4 } & Moderately differentiated & 02 & $10 \%$ \\
\cline { 2 - 4 } & Villoglandular type & 01 & $05 \%$ \\
\cline { 2 - 4 } 2. & Poorly differentiated & 01 & $05 \%$ \\
\cline { 2 - 4 } & Cervical carcinoma & 05 & $25 \%$ \\
\cline { 2 - 4 } & $\begin{array}{c}\text { Well differentiated squamous cell } \\
\text { carcinoma }\end{array}$ & 03 & $15 \%$ \\
\cline { 2 - 4 } & $\begin{array}{c}\text { Moderately differentiated squamous } \\
\text { cell carcinoma }\end{array}$ & 02 & $10 \%$ \\
\hline 3. & Malignant mixed mullerian tumor & 01 & $05 \%$ \\
\hline
\end{tabular}

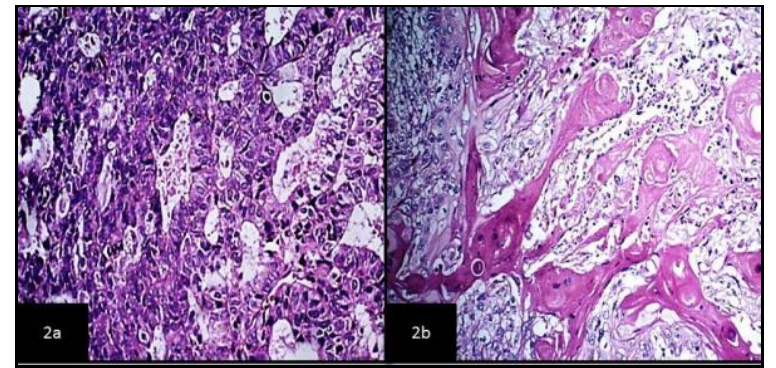

Fig. 2a: Low power view, $\mathrm{H} / \mathrm{E}$ well differentiated endometrial carcinoma

Fig. 2b: Low power view, H/E well differentiated Squamous cell carcinoma

\section{Discussion}

In this era, as life expectancy is increasing women are experiencing a longer postmenopausal phase. PMB is an alarming symptom in this age group although only $10 \%$ of postmenopausal women have PMB ${ }^{4}$ However they have a right to live a long life in good health, rather than one of pain and misery due to PMB. ${ }^{9}$

Postmenopausal bleeding is regarded as an ominous sign of genital pathologies and should always be evaluated promptly. The present study was done to evaluate the spectrum of histopathological changes in PMB and the pattern, incidence of malignant lesions presenting as $\mathrm{PMB}$ in our region.

The duration of menopause before the onset of PMB ranged from 1-26 years and the majority of them presented after 5 years of cessation of normal menstruation. This is in correlation with the studies done by Ubeja et al. ${ }^{4}$
In the present study, the age range of cases was 4184 years. It was observed that maximum numbers of cases were in age groups of 51-60 years.

The mean age in the present study is 58 years. This is in correlation with various other studies ${ }^{4,5,8}$ and is slightly more when compared with other Indian studies. ${ }^{1,10}$ It was also observed that as the age of our subjects increased the incidence of postmenopausal bleeding decreased, indicating an inverse relationship between age and age at which postmenopausal bleeding presented, similar findings were reported by Pragati et al, ${ }^{1}$ Yousaf $S$ et al, ${ }^{12}$ and Gredmark T et al. ${ }^{13}$ The age of occurrence of malignant lesions ranged between 51 years to 80 years.

In the present study, of total lesions, benign lesions accounted for $44 \%$, premalignant lesions $25.7 \%$, malignant lesions $18.34 \%$, and $11 \%$ were reported as inadequate. Similar findings were observed in studies done by Kothapally et al, ${ }^{3}$ Nirupama et al, ${ }^{7}$ Swami et $\mathrm{al},{ }^{14}$ and Usman et al. ${ }^{8}$ But our study is in contrast to the study done by Arati mallick et $\mathrm{al}^{6}$ where malignant lesions were more in number.

In the benign lesions endocervical and endometrial polyps were more in number followed by proliferative type, and atrophy. This is in correlation with the study done by Usman et $a .^{8}$ In the studies done by Sonali et $\mathrm{al}^{5}$ and Arati mallick et $\mathrm{al}^{6}$ cases reported as atrophic were more in number. Bleeding from the atrophic endometrium may be due to unspecified proportion of postmenopausal endometria which, despite being atrophic, retain a weak proliferative pattern for many years, probably as a response to continuous low level oestrogenic stimulation. ${ }^{15}$ 
Endometrial hyperplasia is an oestrogen dependent condition and has the same risk factors as for endometrial carcinoma. The complex atypical hyperplasia has $25-30 \%$ incidence of progression to invasive carcinoma while simple hyperplasia has only $1 \%$ incidence of progression. ${ }^{6}$ In our study out of 28 premalignant lesions 9 cases were simple cystoglandular hyperplasias. Two cases each of simple hyperplasia with focal atypia and complex hyperplasia with focal atypia were also reported. Our findings are similar to the findings by Kothapally et $\mathrm{a}^{3}$ Arati mallick et al. ${ }^{6}$ The risk of malignant transformation increases with increase in complexity and atypia from 1 to $29 \%$ and, therefore, sampling of such lesion becomes mandatory in order to rule out malignancy. ${ }^{8}$ Therefore early detection and proper management has to be offered to such patients. In the present study we had seven cases of cervical intraepithelial neoplasias (CIN), presenting as $\mathrm{PMB}$, which is similar to the findings of Sharma $\mathrm{DD}^{10}$ et al.

Malignancies

In our study we encountered $18.34 \%$ of malignant lesions where in majority were endometrial carcinomas followed by cervical carcinomas and we reported one case of malignant mixed mullerian tumor.

The results from our study emphasise that endometrial carcinoma is the most common malignant cause for postmenopausal bleeding and are comparable with the studies done by Sonali rati etal ${ }^{5}$ and Arati mallick. ${ }^{6}$ But these findings are in contrast to various other Indian studies where carcinoma cervix was more common. ${ }^{1,3,7,10}$ The results of our study are in correlation with the studies done by $\mathrm{Usman}^{8}$ and Bani irshadi. $^{2}$

As our centre caters to urban population, the risk factors associated with urban lifestyle might have contributed for increased incidence of endometrial carcinoma compared to cervical carcinoma.

As there were significant number of even cervical carcinomas in our study, the diagnostic evaluation of PMB in our region should also be focused on cervical pathology apart from endometrial pathology. ${ }^{1}$

\section{Conclusion}

With increased life span the incidence of postmenopausal bleeding is on rise and any bleeding in that age group should be evaluated in the line of malignancy unless otherwise proved. PMB.

A variety of histopathological findings are seen in

Although benign pathologies were more common but the collective proportion of premalignant and malignant conditions is considerably higher in our study.

Thus, postmenopausal bleeding is a symptom of varied etiologies and is not always because of malignant conditions. Therefore, careful histopathologic examination and accurate diagnosis makes it much easier to counsel the patient about further course of management.

Priority should be given at the government level to introduce regular gynaecological screening at different levels of health care for early detection and effective management of PMB. The clinicians should educate the patients about the alarming symptom of PMB and emphasize the need for seeking urgent medical advice and early commencement of treatment.

\section{References}

1. Dr Pragati J Karmarkar, Dr Anne Wilkinson, Dr Mayuri Rathod Histopathological Evaluation of Postmenopausal Bleeding IOSR Journal of Dental and Medical Sciences Volume 13, Issue 10 Ver. III (Oct. 2014), PP 53-57.

2. Bani-Irshaid 1 and A. Al-Sumadi Histological findings in women with postmenopausal bleeding: Jordanian figuresEastern Mediterranean Health Journal Vol. 17 No. 7, 2011.

3. Kothapally K, Bhashyakarla U. Postmenopausal bleeding: clinicopathologic study in a teaching hospital of Andhra Pradesh: IntJ Reprod Contracept Obstet Gynecol. 2013;2(3):344-48.

4. Ubeja A, Singh A. Clinicopathological evaluation of postmenopausal bleeding in rural hospital set up. Int $\mathbf{J}$ Reprod Contracept Obstet Gynecol 2017;6:3556-9.

5. Sonali Rathi, Sangeeta K, Manisha K. "Histopathological evaluation in women with Postmenopausal Bleeding and Associated Risk factors for Endometrial Carcinoma". Journal of Evolution of Medical and Dental Sciences 2013; Vol2, Issue 24, June 17;Page:4397-4402.

6. Arati Mallick, Ritanjali Behera, Khetrabasi Subudhi. "Histopathological study of endometrium in postmenopausal bleeding". Journal of Evolution of Medical and Dental Sciences 2013; Vol. 2, Issue 46, November 18; Page: 9010-9018.

7. Nirupama V, Suneetha Y, Prabha Devi K. PostMenopausal Bleeding: An Analytic Study of 100 Cases. International Journal of Science and Research. 2015:4:2319.

8. Muhammad Usman Tariq, Romana Idrees, Ahmed Raheem and Naila Kayani Spectrum of Histopathological Findings in Postmenopausal Bleeding Journal of the College of Physicians and Surgeons Pakistan 2015, Vol. 25 (11):794-797.

9. Rita D, Sunil Kumar KS, Rithesh SK. The clinicopathological study of postmenopausal bleeding. Int J Reprod Contracept Obstet Gynecol. 2016;5(11):36713674.

10. Sharma DD, Chandnani KA. A study of aetiology and prevalence of malignancy in patients with postmenopausal bleeding. International Journal of Reproduction, Contraception, Obstetrics and Gynecology 2017;6:3973-8.

11. Gandi SR, Vishwekar PS. A study on correlation of Pap smear, colposcopy and colposcopic directed biopsy in women with unhealthy cervix. J. Evolution Med. Dent. Sci. 2017;6(7):515-518.

12. Yousaf S, Shaheen M. and Rana T. Frequency of Endometrial Carcinoma in Patients with Postmenopausal Bleeding. ANNALS 2010;16 290-94.

13. Gredmark T, Sonja Kvint, Guillaume Havel, Lars Ake Maltsson. Histopathological findings in women with 
postmenopausal bleeding. British Journal of Obstetrics and Gynaecology 1995;102:133-36.

14. M.Y. Swami, M.B. Swami, Histopathological evaluation of endometrium in pre and postmenopausal uterine bleeding. Indian Journal of Obstetrics and Gynaecology Research 2015;2(4):264-269.

15. E Sivridis, A Giatromanolaki Proliferative activity in postmenopausal endometrium: the lurking potential for giving rise to an endometrial adenocarcinoma J Clin Pathol 2004;57:840-844. 